\title{
浅析印尼建国大学中文系学生学习汉语时常犯的错误
}

\section{THE ANALYSIS OF COMMON MISTAKES MADE BY CHINESE DEPARTMENT STUDENTS OF BINUS UNIVERSITY IN LEARNING MANDARIN}

\author{
Andyni Khosasih \\ Chinese Department, Language and Culture Faculty, BINUS University \\ Jl. Kemanggisan Ilir III No 45, Kemanggisan, Jakarta Barat 11480 \\ Andyni_Khosasih@binus.edu
}

\begin{abstract}
Article was presented in two sections, those were general overview of Chinese Department of Binus University and common mistakes made by students in learning Mandarin. To find the data, a direct observation was done in the classrooms. It can be concluded that in learning Mandarin, students' mistakes are caused by the influence of using the mother tongue, the laziness of students in learning the language, and the lack of basic Mandarin knowledge. Common mistakes made by the students in language skills area are pronouncing vowels and consonants, intonation, grammar, Hanzi characters, and vocabularies.
\end{abstract}

Keywords: Chinese students, learning, Mandarin, common mistakes

\section{内容提要}

现在有 400 多名学生在建大中文系学习汉语。在学习汉语的过程中, 他们常犯一些错误。 为了了解三年级学生学习汉语时经常犯的错误, 笔者选择 “浅析印尼建国大学中文系学生学习 汉语时常犯的错误” 作为论文题目。此论文主要可分成两个部分：一、印尼建国大学中文系简 介; 二、建大中文系学生学习汉语时常犯的错误。笔者使用的调查方式是现场调查。通过调查 与分析后, 笔者得出的结论是建大中文系学生在学习汉语的过程中常犯错误是因为: 一、受到 母语的影响; 二、学生在学习过程中不太认真; 三、学生没有良好汉语基础。学生学习汉语时 常犯的错误是声母和韵母、声调、语法、汉字与词汇。

关键词: 中文系学生学习汉语 错误 


\section{前言}

随着中国经济的发展, 目前许多人都在学习汉语。对一些使用拉丁字母的国家来说, 要掌 握好汉语是一件不容易之事。印尼学生学习汉语时经常觉得汉语很难。印尼建国大学（简称建 大）中文系学生也有同样的看法。建大中文系学生自一年级开始就学习语法、口语、听力与读 写课。尽管如此, 建大中文系学生在学习汉语时还经常犯错误。为了了解学生学习汉语时经常 犯的错误, 笔者选择 “浅析印尼建国大学中文系学生学习汉语时常犯的错误” 作为论文题目。

\section{浅析印尼建国大学中文系学生学习汉语时常犯的错误}

\section{一、印尼建国大学中文系简介}

随着汉语热, 学习汉语的学者越来越多。男女老幼无论在正规学校或非正规学校（例如: 补习班、家教）学习汉语。目前几乎所有的私利学校开办中文课。现在在某些学校, 中文课已 成为必修课。除了学校开办中文课以外, 如今印尼的大学开办中文系的日益增加。比如：雅加 达、泗水、日惹、万隆、棉兰等城市。在雅加达, 设有中文系的大学共有 8 所, 其中是印尼建 国大学 (Universitas Bina Nusantara)、印尼大学 (Universitas Indonesia)、新雅学院 (Xinya College) 、印尼基督教大学 (Universitas Kristen Indonesia) 、圣母大学 (Universitas Bunda Mulia) 、阿尔·哈沙（回教）大学 (Universitas Al Azhar Indonesia）、达尔玛勃沙塔大学（Universitas Darma Persada）与国民大学（Universitas Nasiona1）。基本上这 8 所大学可分成两类: 国立大学和私立大学。印尼建国大学属于私立大 学的性质。

建大中文系现在是印度尼西亚最好的中文系之一。在印尼，迄今绝大多数中文系只有得到 “及格” 的本科评估成绩。从十几所大学当中, 只有两所大学拥有 “良” 的本科评估成绩。建 大中文系是其中一个，另外一所是印尼大学。

印尼建国大学中文系建立于 2002 年, 至今已有六届毕业生。目前有 4 个专业, 其中是经 贸专业, 文化专业, 传媒专业和教育专业。学生可选择自己所意愿的专业方向。从 4 专业方向 当中，经贸专业最受欢迎。建大中文系目前共有 33 位教师，419 名学生。从 33 位教师中， 27 位是本地教师，6 位中国教师。8 位教师 (24.2\%) 的专业背景是非中文系或汉语言专业。这些 教师主要是专业人士。他们都是公司的老板或领导。这些教师所教的科目是经贸或传媒专业。 上课时都以中文授课。

\section{二、建大中文系学生学习汉语时常犯的错误。}

建大中文系学生主要可分成两类: 非华裔学生和华裔学生。非华裔学生绝大多数 来自雅加达。他们基本上都没有汉语基础。可说是从零起点开始学习汉语的。因没有 汉语基础的缘故, 所以在学习过程中不少学生遇到困难。华裔学生大多数也是来自雅加达。除 
了来自雅加达以外, 少数学生来自邦加、巨港、坤甸、巴干西阿比阿比、占碑。华裔学生大都 已有汉语基础。有的自幼就开始学习中文, 有的是在补习班或在学校学习的。少部分是父母教 的。虽然已有汉语基础, 但大都学生的汉语基础都不太巩固。外地学生的汉语基础比本地学生 或来自雅加达的华裔学生好。华裔学生虽然绝大多数已有汉语基础, 可在学习上还是经常犯同 样的错误。

$$
\text { （一）声母与韵母。 }
$$

初学者学习汉语时首先得学好汉语拼音。掌握汉语拼音之前必须先掌握好生母和韵母。建 大中文系学生学习汉语时经常没有掌握好声母和韵母。主要原因是学生们不重视声母和韵母。 许多学生认为掌握好汉语不需要掌握好声母和韵母因为不影响他们的中文水平。因此学生们在 学习声母和韵母时经常忽略声母、韵母的发音部位和发音方法。声母的不同是由发音部位和发 音方法不同决定的。韵母也是如此。他们认为只要对象能听懂他们所想表达的意思就足够了。 另外, 有些学生不掌握声母和韵母的基本知识。他们总是分不清楚如何准确地发出声母和韵 母。以下是学生们经常犯的错误:

1. “b”、“p”、“d” “t”、“g”、“k”。

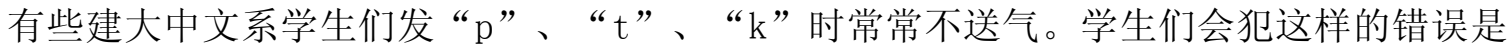
因为在印尼语里发声母时不分送气和不送气。“ $p$ ” 经常发成 “b”、“t”常常发成 “d”、而 “k” 却发成 “g”。例如: “pai” 发成 “bai”、“ta” 发成 “ da”、“ke” 发成 “ge”。建大中文系学生发 “ $p$ ”、“ $t$ ”、“ $k$ ” 时经常使用拉丁字母的方法。这导 致虽然有些学生已是三年级的学生, 但他们在发这三个声母时还是常常犯错误。另外, 建 大中文系学生发 “b”、“d”、“g” 时绝大多数准确, 原因是 “b”、“d”、“g”与印 尼语里的“p”、“ $\mathrm{t} ” 、 “ \mathrm{k} ”$ 发音方法一样。

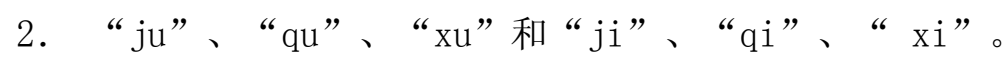
学生们常常分不清出 “ ju”、“qu”、“xu” 和“ $\mathrm{ji}$ ”、“qi”、“ $\mathrm{xi}$ ” 的发音部位和

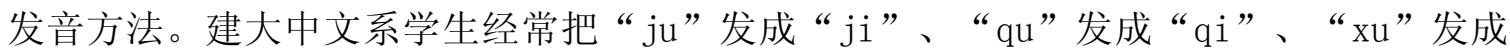
“xi”。主要原因是 “ $\mathrm{j}$ ” “ $\mathrm{q}$ ” “ $\mathrm{x}$ ”的发音方法与印尼语不一样。另外, “ $u$ ”在印尼 语的发音方法和汉语大大不同。这导致学生在这方面常常犯错误。有些学生虽然知道他们 所发的声母或韵母不准确, 可也不想纠正因为他们认为只要对方听得懂他们所表达的意思 就足够了。

3. “zhi”、“chi”、“shi”、“ ri”。

汉语的“zhi”、“chi”、“shi”、“ 没有圈舍的方法。因此, 发 “zhi”、“chi”、“shi”、“ ri” 时, 许多学生都不准 确。另外, “ $i ”$ 只发 “ $i ”$, 而在汉语 “ $i$ ”可发成 “ $i$ ” 和 “- $i ” 。$

4. “ao” 和 “ian”。

少部分学生在发 “ao” 和 “ian” 时经常犯错误。比如: “hao” 发成 “hoa”。有的学生 在发 “ian” 时是根据印尼语的方法。一般本地学生才会出现这个错误。通常学生会犯这 个错误的原因是因为学习不认真。 
从以上分析, 笔者得知学生在声母和韵母方面经常犯错误的主要因素是因为受到母语的影响。 其他原因是学习时不太注意如何准确的发声母和韵母。有些学生会犯错误是因为学习不认真。

$$
\text { (二) 声调。 }
$$

声调是汉语的特点。许多国家在他们的语言里都没有声调, 其中是印度尼西亚。普通话的 声调可分成四类：一、阴平 (第一声)；二、阳平 (第二声)；三、上声（第三声）；四、去 声 (第四声)。建大中文系学生掌握声调时经常遇到困难。从四个声调当中, 有些学生分不清 楚阳声与上声。因此，与阴声、去声对比，阳声、上声的错误更多。虽然学生在阴声、去声的 错误较少, 可有少数学生却无法分别阴声和去声。他们把阴声发成去声或把去声发成阴声。阳 声与去声也是如此。除了这些错误, 因不掌握声调的变调的缘故, 所以在这方面所犯的错误更 多。

下面是学生经常犯的错误:

1.“很难”。“很”在此该发上声，可建大中文系学生有时却发成阴平。

2. “可以”。“可” 在这里该发阴平，但却发成上声。

3. “我很好”。在此有的学生把 “我” 发成, 而不是上声, 把 “很” 发成上声（第三声）。

“好” 字的声调基本上都准确。

4. “哪” 和 “那”。建大中文系学生常常把 “哪” 发成去声, 把 “那” 发成上声。他们会犯 这样 的错误是因为分不清楚 “哪” 和 “那” 的用法。

5. “高兴”。学生们经常把 “高”发成去声。

\section{（三）语法。}

语法对一个学习汉语的学者来说经常成为掌握汉语的一个障碍。建大中文系学生也面对同 样的困难。原因是印尼语法与汉语的语法不一样。例如: 我在建国大学学习汉语。此句根据印 尼语里的标准语法是 “我学习汉语在建国大学”。因此在日常生活中, 学生经常认为 “我学习 汉语在建国大学” 是正确的句子。另外, 学生在语法方面常出现的错误是所使用的声词不准 确。

\section{（四）汉子。}

汉字是外国人学习汉语时不易掌握的技能。印尼使用拉丁字母, 所以掌握汉字对印尼学生 来说（包括建大中文系学生）是一件不容易的事情。少部分建大中文系学生完全没有汉语基 础。因此, 汉字对这些学生来说很陌生。若要掌握汉字得下不少功夫。建大中文系学生难以掌 握汉字是因为他们不常练写汉字。有些中文系学生不掌握笔顺、笔画、部首。少部分学生不知 道笔顺、笔画和部首。如：学生写 “宿” 字的时候把 “点” 写成 “坚”。其它例子是学生说不 出汉字的部首。这导致学生难以巩固地掌握所学过的汉字。另外, 教师在教学过程中常常发现 学生记不住汉字，如：“我” 写成 “找”。华裔学生在掌握汉字方面比本地学生强。汉字对大 多数华裔学生来说不陌生。虽然进入中文系之前不会写汉字或没有汉语基础, 但在家里、亲戚 家、或在社会上基本上都接触过汉子。这是与本地学生的区别。本地学生大都进入中文系之后 才认识汉字。这样, 华裔学生在掌握汉字时比本地学生好。尽管如此, 华裔学生掌握汉字时所 面临的困难与本地学生一样。 
（五）词汇。

词汇又称语汇, 是一种语言里所有的 (或特定范围的) 词和固定短语的总合。词汇是语言 的建筑材料, 没有建筑材料就不能盖房子, 没有词汇就不能造句子。语言是用一个一个词按照 有关的语法规则组合起来造出种种句子进行交际的。对一个人来说, 他掌握的词越多, 他的词 汇就越丰富, 也就能越确切的表达意思。积累词汇的方法是: 一、深入生活, 在丰富的语言生 活中有意识的搜集、记录各种类型的词汇; 二、认真阅读古代的、现代的、文艺的、政论 的、科技的各种作品, 从中吸取语言营养; 三、加强写作实践, 熟悉, 掌握各种类型的词汇。

不少建大中文系学生在词汇方面比较弱。大都学生掌握的词汇量不多。主要原因是学生们 极少复习所学过的生词。其他原因是他们也极少做练习或者在课堂上不听教师所讲的课, 甚至 少部分学生常常旷课。这样学生们在做词语搭配的练习时经常发生错误。因为掌握的词汇量太 少, 所以当教师布置词语搭配的练习时, 这些学生无法准确的回答问题。不掌握良好的词语搭 配技能导致学生们在造句时经常遇到困难。学生们不知道该如何正确地表达他们的想法。这 样无法与周围的人们进行交际。

\section{结论}

从以上分析笔者得出的结论是建大中文系学生在学习汉语的过程中常犯错误是因为: 一、 受到母语的影响; 二、学生在学习过程中不太认真; 三、学生没有良好汉语基础。学生学习汉 语时常犯的错误是生母和韵母、声调、语法、汉字与词汇。

\section{参考文献}

1. 中原大学应用华语文学系・印尼华文教育语教学【M】・中原大学应用华语文学系出版, 2006 年

2. 黄伯荣、廖序东·现代汉语增订三版上册【M】・高等教育出版社, 2001 年

3. 黄伯荣、廖序东·现代汉语增订三版下册【M】·高等教育出版社, 2001 年

4. 李德津、程美珍·外国人使用汉语语法【M】·华语教学出版社，2003 年

\footnotetext{
1 黄伯荣、廖序东 现代汉语增订三版上册【M】 高等教育出版社, 2001 年, 216 页
} 